\title{
Cambios de código en niños mientras juegan videojuegos
}

\author{
Armando Robles Hmilowicz, Universidad de Buenos Aires, Argentina
}

\section{Introducción}

La capacidad de hablar más de una lengua es algo habitual entre la mayoría de los habitantes del mundo. Sin embargo, los primeros trabajos sobre bilingüismo presuponían que el hablante monolingüe era la norma. Este punto de vista supone que los hablantes bilingües cuentan con “two separate and isolatable language competencies” (Grosjean 1989: 4) semejantes a las de dos hablantes monolingües distintos. Así, el uso o mezcla de dos códigos por parte de un hablante se equiparaba con una falta de cuidado, una interferencia o una discapacidad para distinguir entre dos lenguas.

El empleo de más de una lengua durante una conversación, fenómeno conocido como code-switching (en adelante, CS), es observado cada vez con mayor frecuencia dado el crecimiento en el número y variedad de interacciones humanas, resultado de una mayor movilidad social y económica y de los avances en los medios técnicos de comunicación. Constituye además un fenómeno complejo y variable, difícil de categorizar y predecir, al surgir cuestiones técnicas como la definición de una 'lengua base' (Mondada 2007; Nussbaum y Tusón 2003; Álvarez-Caccamo 1998) y la combinación de códigos o codemixing, fenómenos que demuestran que las fronteras entre una lengua y otra no siempre son claras.

Distintos autores, entre los que destacan Hymes (1972, con Gumperz), Gumperz (1982) y Auer (1984), han destacado la capacidad de los hablantes de cambiar de código durante distintos momentos de la conversación, ya sea desde una perspectiva etnográfica o desde la sociolingüística y el análisis interaccional. La mayor parte de estos estudios se han dedicado a describir esta conducta entre interlocutores adultos; sin embargo, desde hace algunos años también se han dedicado esfuerzos a estudiar el CS entre los niños, en especial en situaciones estructuradas como el salón de clases de primaria y secundaria (Unamuno 2008, 2004; Nussbaum 2001) o en centros de aprendizaje de lenguas. 
Se ha señalado que los cambios de código que tienen lugar en las aulas entre hablantes bilingües o plurilingües representan distintas estrategias en distintos contextos comunicativos, tanto endolingües como exolingües (entre hablantes con competencias simétricas o asimétricas, respectivamente) (Lüdi \& Py 2003). Pero hace falta extender el estudio de este fenómeno a otros contextos a fin de obtener una visión más integral de sus alcances y limitaciones y entender cómo operan como recursos. Por ejemplo, se ha prestado menos atención a los cambios de lengua entre pares, y la mayoría de los datos de interacción entre niños bilingües proviene normalmente de contextos institucionales. En este sentido un aporte interesante viene de Cromdal (2005), quien en un contexto escolar describe el CS entre niños mediado a través de una computadora. También existen algunos estudios sobre el CS en contextos de juego (Bauer, Hall y Kruth 2002), pero, como veremos más adelante, son los menos.

Aquí intentamos presentar un contexto un tanto diferente. Se trata de una descripción del CS que tiene lugar también entre niños, pero en una situación que, si bien tiene cierta estructura, es indudablemente más informal: el juego entre niños en un contexto vacacional, en particular durante una sesión de juegos de vídeo. Los niños de nuestro caso de estudio son una pareja de hermanos nacidos en Estados Unidos, de padres mexicanos. Son bilingües y hacen uso corriente del CS entre el inglés y el español, parte de lo que en su entorno se conoce como spanglish (Ardila 2005). El spanglish es la mezcla del inglés y el español observada entre las comunidades hispanas o latinas de los Estados Unidos, y constituye una de las manifestaciones lingüísticas actuales más importantes en aquel país. Representa un fenómeno muy extendido y que presenta numerosas variaciones dependiendo de la región. Se han descrito dos tipos: uno superficial, que incluye préstamos y CS, y otro profundo con fenómenos léxico-semánticos y gramaticales más complejos.

El trabajo aplica el enfoque metodológico del análisis interaccional a fin de describir los cambios de código que tienen lugar entre estos hermanos y en ocasiones con sus parientes mexicanos. Mediante el análisis de las interacciones buscamos identificar bajo qué circunstancias surgen los cambios de código a fin de encontrar algunos patrones 
de uso y también buscamos estudiar la manera en que aprovechan este recurso lingüístico desde el punto de vista comunicativo al momento de realizar una tarea, en este caso lúdica.

\section{Problema}

Como veremos más adelante, los principales estudiosos del CS han propuesto algunas categorías para clasificar este fenómeno. Una de las divisiones más importantes fue propuesta por Gumperz (1982), quien distinguió entre code-switching situacional y codeswitching metafórico.

En nuestro caso queremos evaluar si resulta posible hacer una analogía entre estas categorías y un par de categorías que hemos adaptado al caso concreto de estudio. Así, nuestro problema consiste en determinar si las categorías de CS de orientación externa (cuestiones no relacionadas con el videojuego) y CS de orientación interna (cuestiones relacionadas con el videojuego) resultan útiles para describir las sesiones de juego que analizamos.

Además estudiaremos el papel que ejercen los cambios de lengua en el ámbito de los videojuegos como recurso comunicativo, buscando determinar si en efecto constituyen un recurso en este contexto.

\section{CS en el aula y en el juego}

El CS en general ha sido objeto de numerosas investigaciones en las últimas décadas. Según Mondada (2007), el CS suele registrarse en contextos socioculturales caracterizados por el contacto entre distintas lenguas debido a la movilidad de los locutores y a cuestiones identitarias y sociales expresadas a través de prácticas lingüísticas. Además, desde un punto de vista sumamente crítico, describe una articulación entre lengua, sociedad, cultura y contexto, y menciona distintas formas en que se ha pensado dicha articulación: como reflejo de estructuras sociales, ideologías, identidades, conflictos, etc.; como correlación entre cierto acto de habla y alguna variable (discurso privado vs. público, tipo de interlocutores, discurso referido, etc.); entre otras. Sin embargo, explica que estos modelos 
sólo pueden dar cuenta de tendencias generales, pero poco pueden decirnos sobre los detalles del CS que observamos en la interacción. Por ello la autora destaca la necesidad de un enfoque interaccional que considere el CS como un recurso empleado por los participantes de manera contingente, situado localmente y sensible a la organización secuencial de las interacciones en curso.

Mencionamos en la Introducción que se han abordado estudios de CS desde el aula y desde el juego, aunque en diferente proporción. A continuación haremos un breve repaso de lo más relevante que han aportado estas dos perspectivas al estudio del CS.

El CS en el aula: El estudio del CS en las aulas se ha centrado en el papel de los cambios de lengua en la reestructuración de los repertorios lingüísticos y comunicativos de los participantes. Por tanto, el CS se ha conceptualizado como un recurso que permite detectar marcadores de adquisición, selección y uso de conocimientos nuevos en el aula (Lüdi y Py 2009).

Hemos mencionado ya la distinción propuesta por Gumperz entre dos tipos de CS: el CS situacional (que tiene lugar por razones extralingüísticas) y el CS metafórico (motivado por razones inherentes al discurso o interacción en curso). A esta distinción se agrega la que expuso Auer en 1984. Auer habla de CS relacionado con el participante y CS relacionado con el discurso. El primero tiene que ver con la competencia o incompetencia del hablante, mientras que el segundo con la construcción de actividades interactivas. En contextos de aprendizaje de lengua, el hablante que ponga en escena el CS relacionado con el participante tenderá a usar la lengua que mejor domine para evitar equivocaciones, utilizará determinado idioma por limitaciones institucionales o cuestiones de actitud, o elegirá emplear la lengua de su interlocutor para facilitar la comprensión durante la interacción. Por otra parte, el CS relacionado con el discurso guarda relación con la organización de la interacción, ya que contribuye a ésta otorgando significado a los enunciados. Así, en contextos de enseñanza-aprendizaje de lenguas, el CS puede ser indicador de algún cambio en los parámetros de interacción; por ejemplo, un cambio de tema, la entrada de un nuevo interlocutor, la introducción de una nueva "voz" en un enunciado determinado, discurso referido, un chiste, etc. 
Autores como Liebscher y Dailey-O’Cain (2005) y Zentella (1981) plantean que la forma de CS más común en el aula es la relacionada con el participante. Ello se puede deber a distintos motivos, como la incapacidad de un estudiante para expresarse o la intención del profesorado de brindar ayuda. Sin embargo, en el aula también es posible ver ejemplos de CS relacionado con el discurso (Unamuno 2008).

El CS en el juego: En el contexto del juego debemos destacar un caso de estudio llevado a cabo por Bauer y colaboradoras (2002) desde una perspectiva interaccional y funcional. Esta autora también se pregunta por el papel que desempeña el CS en las interacciones de niños bilingües y la forma en que desarrollan sus habilidades comunicativas (aunque en su caso estudia una interacción niño-adulto). Parte de Nicoladis y Genesee (1996), quienes afirman que se puede estudiar esta cuestión desde dos perspectivas. Por un lado, existen quienes ven detrás del CS las necesidades cognitivas de los niños, y concluyen que éstos alternan de lengua de manera estratégica, cambiando de un código a otro cuando no saben cómo decir algo en determinada lengua o cuando no cuentan con una traducción directa a la mano. Por otro lado, los niños bilingües suelen cambiar de código al usar la lengua con la que se sienten menos seguros. Bauer (2000) también defiende este planteo sugiriendo que los niños bilingües cambian de código de manera sistemática dependiendo de cómo encaran determinadas tareas de lectura. Otra forma de ver el problema, adoptada por Bauer y colaboradoras en su artículo, es desde una óptica interpersonal o social. Con base en un trabajo de Genesee, Nicoladis y Paradis (1995), sugieren que los niños cambian de código según perciben cuál es la lengua que prefiere su interlocutor, mostrando cierta sensibilidad a las normas y expectativas socioculturales de sus contextos comunicativos.

Sobre estas bases, Bauer y colaboradoras (2002) describen las distintas formas en que la niña de su caso de estudio utiliza las dos lenguas que conoce, el inglés y el alemán, mientras juega con sus padres y su cuidadora. En la primera parte de su estudio, Bauer et al. analizan los tipos de juego que desarrolla la niña con sus interlocutores, y el resultado es una triple división: juegos dirigidos por la niña, juegos dirigidos por el adulto y juegos de “rol compartido”. Ello le permitió abordar su segunda pregunta, más emparentada con 
el fenómeno del CS: ¿Cómo utiliza la niña sus dos lenguas para constituir su participación en el juego? El estudio reveló que los tres tipos de actividad le brindan a la niña la oportunidad de utilizar el lenguaje de maneras distintas para llevar a cabo tipos similares de juego. Mediante el análisis de las interacciones, se detectaron algunas diferencias sutiles en su uso del lenguaje, tanto en inglés como en alemán. Por ejemplo, cuando la niña es quien dirige la actividad, tiende sólo a utilizar el inglés. Lo más interesante es que, por medio de sus interacciones con distintos interlocutores, la niña aprende y ensaya distintas habilidades comunicativas en ambos idiomas. De modo particular, según Bauer, la niña aprende cómo la lengua que elige siempre ejerce un efecto sobre las acciones de sus interlocutores y sobre lo que pretende que sepan y entiendan.

A fin de estudiar el CS entre niños en el contexto de los videojuegos, debemos llevar a cabo un análisis detallado de las interacciones de los participantes, centrándonos en cómo usan sus repertorios lingüísticos, cómo aprovechan las diferencias que detectan en las lenguas que usan y cómo explotan su combinación. Para ello, siguiendo a Unamuno (2008), hemos adoptado un enfoque cualitativo con el fin de estudiar interacciones bilingües en las que la organización ejerce un papel importante. Esta visión supone que el momento en que tiene lugar el CS y la naturaleza de la actividad comunicativa realizada en este caso el juego- son importantes para comprender este fenómeno lingüístico. En otras palabras, no se puede ver el CS como algo abstracto, que siempre ocurre por los mismos motivos. Debemos ir a la interacción para entender cuándo, cómo y por qué ocurre.

\section{Corpus y metodología}

Contexto del estudio: Los datos analizados provienen de un conjunto de videograbaciones que muestran las vacaciones de una familia de origen mexicano (padre, madre, dos niños) que vive desde hace diez años en Estados Unidos. Las grabaciones tuvieron lugar en diciembre de 2009 al norte de México. Los niños nacieron en Estados Unidos y son bilingües. Los datos utilizados en este artículo provienen de dos sesiones de juego, una en 
la que sólo se encuentran los dos hermanos en cuestión, y otra en la que también participan dos primos de edades similares.

Participantes clave:

- Ángel: hijo mayor, nacido en Estados Unidos, de 8 años de edad. Aprendió primero el inglés y luego el español en la escuela. Habla predominantemente en inglés, incluso con sus padres.

- Christopher: hijo menor, nacido en Estados Unidos, de 4 años de edad. Aprendió inglés y español al mismo tiempo en casa. A diferencia de su hermano, empezó hablando predominantemente español, aunque ahora interactúa en ambas lenguas.

Otros miembros de la familia ampliada que participaron de alguna manera en el estudio son:

- Oscar: primo de Ángel y Chris, mexicano, de 10 años de edad. Hablante nativo del español, sin conocimientos de inglés.

- Víctor: hermano de Oscar, mexicano, de 6 años de edad. Hablante nativo del español, actualmente estudia inglés en la escuela primaria tres veces por semana y dispone de un repertorio básico.

- Raquel: tía de los niños, encargada de realizar las grabaciones e interactuar con los niños. Es bilingüe secuencial pero convive con sus sobrinos en español.

Contexto lingüístico: Según reporta la familia, en su casa en EE.UU. los niños hablan en inglés si están solos o con la madre (salvo que la madre necesite imperiosamente de su atención), y en español si están con el padre o algún otro pariente; en la calle utilizan ambas lenguas. De visita con su familia en México, los niños hablan predominantemente en español, en especial si están con sus primos o tíos, aunque si se encuentran solos suelen hablar en inglés. Ello se debe en buena medida a una consigna explícita emitida por la madre: NO hablar en inglés cuando estén en compañía de otras personas para que no se 
sientan excluidas. En general, Ángel se apega de manera bastante estricta a la regla, recordándole a Chris en repetidas ocasiones la consigna. Todo lo anterior nos dice que, según la situación, los idiomas empleados por los participantes del estudio pueden variar.

Recopilación de los datos: La recolección de datos tuvo lugar en dos sesiones de juego distintas en dos días distintos del viaje vacacional de la familia en cuestión. En la primera sesión, sólo Ángel y Chris aparecen jugando juegos de vídeo (el sistema portátil Nintendo DS) en un sillón (ver Figura 1).

Figura 1. Primera sesión de juego

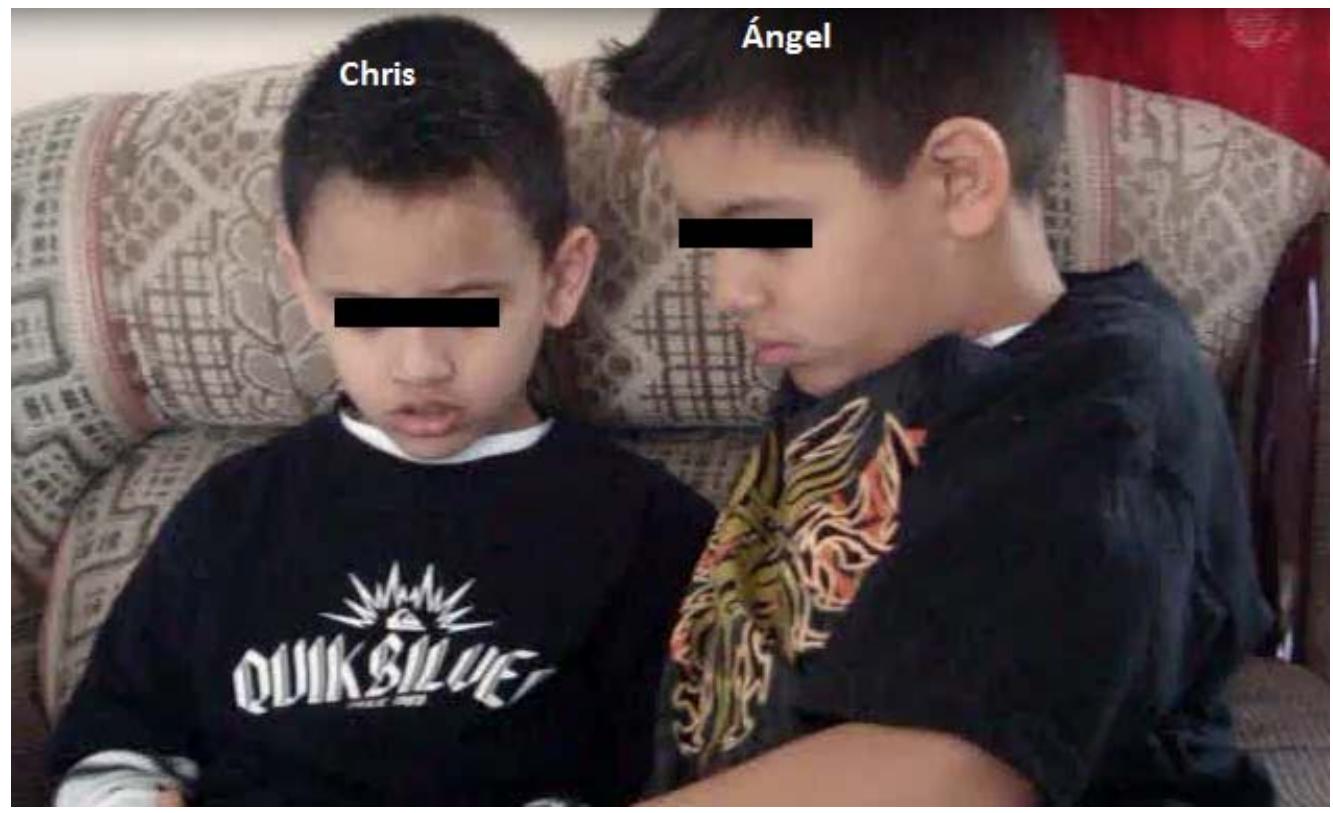

En la segunda sesión, además de Ángel y Chris, participan otros parientes: los primos Oscar y Víctor (Figura 2). 
Figura 2. Segunda sesión de juego

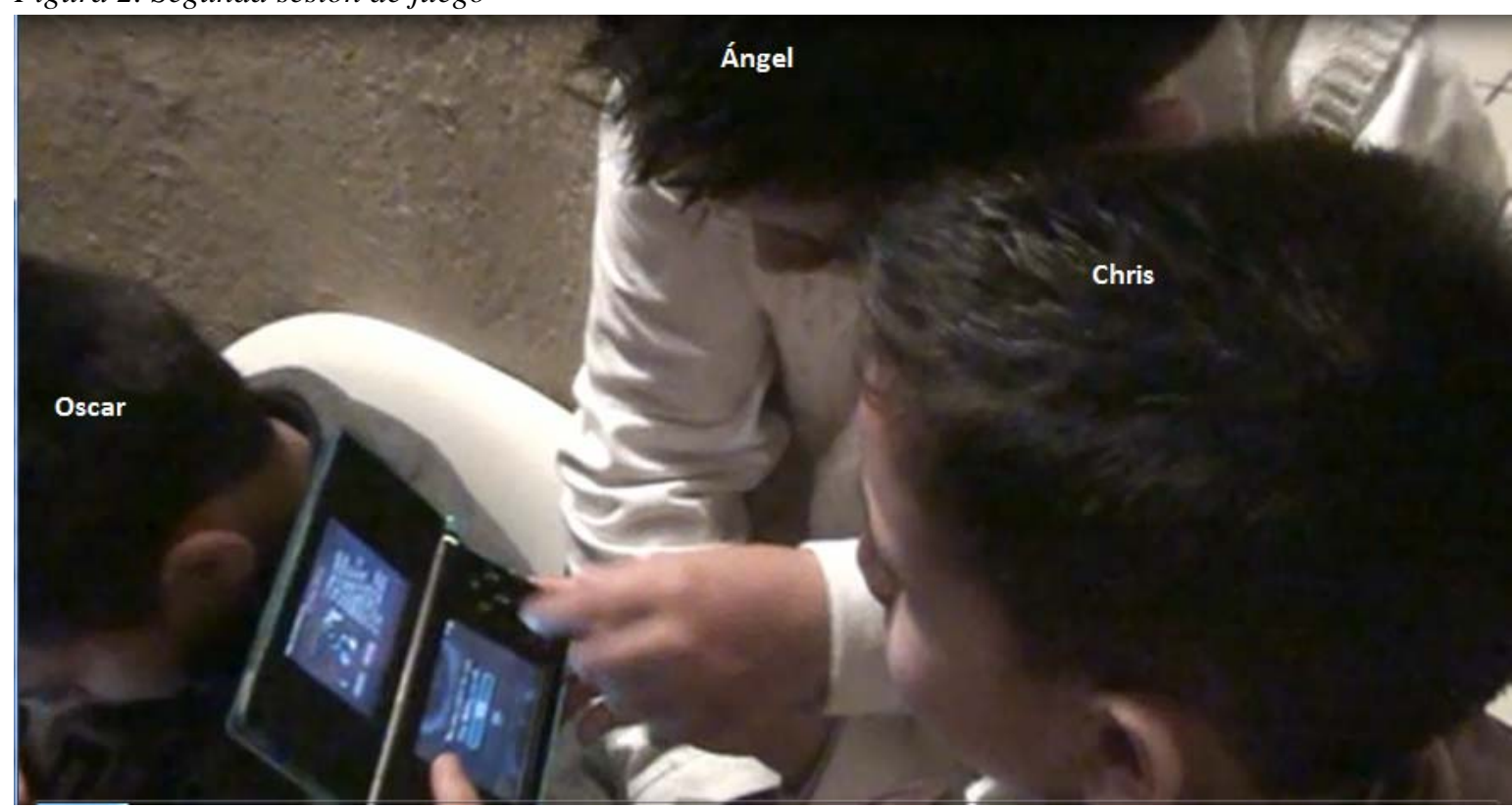

En ningún caso se ofrecieron indicaciones a los niños en relación con el estudio, simplemente se iban registrando las distintas secuencias de juego en las que participaban; tampoco recibieron información de antemano sobre el motivo de las grabaciones. Se obtuvieron 16 vídeos con una duración total de 28 minutos 45 segundos. Las grabaciones se enfocaron a secuencias de juego o de interacción concretas.

A partir de los 16 vídeos se transcribieron los fragmentos en los que el fenómeno de CS era más evidente, aunque el resto también fue analizado por motivos de comparación y para registrar los idiomas utilizados en cada interacción. La transcripción fue llevada a cabo siguiendo las convenciones descritas en el Apéndice 1.

\section{Análisis y resultados}

Tras observar los vídeos y las transcripciones, identificamos casos de code-switching ocurridos durante las secuencias de juego. Primero analizamos los casos de la primera sesión de juegos, en la que sólo participan Ángel y Chris, y cuya lengua base o matriz, según los datos, es el inglés (con cambios al español). Posteriormente analizamos la 
segunda sesión, en la que participan además Oscar y Víctor, y cuya lengua base es el español.

¿Cómo utilizan los interlocutores bilingües de nuestro caso los CS? En el apartado de Problema propusimos la existencia de dos tipos de actividades cuya diferenciación es señalada por el cambio de códigos en el contexto de los juegos de vídeo: los CS de orientación interna (relacionados con el videojuego) y los CS de orientación externa (no relacionados con el videojuego). Desarrollamos estas categorías porque, tal y como lo han mostrado los sociolingüistas interaccionales, el cambio de lengua actúa como un elemento que indexaliza cambios en la situación de interacción (ya sean en el juego o fuera de él), y porque a su vez dichos cambios pueden estar directamente relacionados con el cambio de lengua. Cuando decimos “cambios de orientación interna” u orientados hacia el juego nos referimos a los cambios relacionados con los aspectos intangibles o virtuales del juego (eventos, personajes, ganadores, locaciones, reglas, marcadores de resultado, etc.). Ello contrasta con los cambios de orientación externa, entendiendo por lo “externo” todo lo que sucede en el entorno de la interacción (lo situacional, en términos de Gumperz), incluyendo los aspectos físicos del videojuego, como los cartuchos o la consola misma, que propiamente no forman parte de lo que consideramos el juego.

Originalmente ideamos las categorías teniendo en mente los casos en que la lengua base fuera el español, ya que, al ser producidos en inglés la mayoría de los juegos de vídeo, pensábamos que los diálogos y el vocabulario de los juegos darían lugar a cambios de orientación interna del español al inglés. Sin embargo descubrimos que las categorías también resultaban útiles en el caso contrario, cuando la lengua base es el inglés, como sucede en la primera sesión de juegos, en la que sólo participan Ángel y Chris. Hemos marcado en negritas las enunciaciones realizadas en español para distinguirlas de las enunciaciones inglesas.

\section{Fragmento $1^{\text {i }}$}

1. $\quad$ C - Angel | Angel how did he beat me/ | I pass:ed | I had a like a thousand ten (2)

2. A - yo también (2) 
3. $\mathrm{C}-$ yeah $\mid$ Angel, when I was like over that like don don don don =don don=

4. $\quad \mathrm{A}-=$ es que la verdad $=$ te estoy pidiendo perdón (4) porque yo ya estaba perdonado (XXX) ((voz muy baja))

5. C - no Ángel

6. A - @ (14)

7. $\mathrm{C}$ - why don't/ | Angel Angel (XXX) level eight | right/

8. $\quad A-$ es a diez (2)

9. $\quad \mathrm{C}-\mathrm{C} U|\mathrm{~S}| \mathrm{P} \mid$ eh $\mid$ eja $\mid$ eh eh a::::h eja | stand (3) why/ (4) uh oh my goodness (3) y hay otro/ (3) no no está otro, verdá/

10. A - sí alright | he's gonna be right

11. $\mathrm{C}-$ yeah $\mid$ two $\mid$ ok you won them (2)

12. A $-(\mathrm{XXX})$ careful it's again into $(\mathrm{XXX})$

13. C - Jan and Mark/

14. A- yeah/

15. $\quad \mathrm{C}-$ oh yeah/ $\mid$ me either $\mid$ Jan and Mark | Angel | fiuf

16. A - I drove away from (XXX) (4) O:::h he wins/ | everything does a (XXX) e:::h (5) (XXX)

17. C - yeah

18. A - I was gonna (XXX) ((voz baja))

19. $\mathrm{C}$ - Angel I drik did a krikle K-O

20. A - good (XXX)

21. $\mathrm{C}=\mathrm{I}$ did/ $\mid$ but I couldn't do it $\mid$ ahh/ $/(\mathrm{XXX})$ eehh/ it go a:::::h (XXX)

22. A - ah se apagó

Tal y como muestra este fragmento, si bien en los primeros turnos hay una negociación de la lengua en que llevarán a cabo la actividad, el cambio del inglés al español sitúa los enunciados fuera del marco del juego (en este caso Mario Kart). Así, temas como la petición de perdón de Ángel a Chris o la descripción del hecho (físico) de que se apagó la consola del juego motivan un cambio al español. Por otro lado, cuestiones como el nivel de juego en el que está uno de los interlocutores, quién le ha ganado a quién, logros o records personales, o con qué personaje se tiene que interactuar en el transcurso del juego, parecen motivar que se regrese al inglés o que se mantenga la conversación en esta lengua, tanto en el caso de Ángel como en el de Chris.

Sin embargo, el segundo fragmento que presentamos parece convalidar la tendencia del papel del CS en la interacción con videojuegos, si bien sólo es un fragmento muy breve y en el que sólo se presenta un caso de CS: 


\section{Fragmento 2}

1. C - Ángel ya el mío | sí prende (3) el mío está cargad... ((se sube a un sillón)) why you don’t wanna fight 'em।

2. A - because (2)

3. C - hand on hand/ (7) I wanna be second / (XXX) right/ (4) oh/ look him/

4. A-chops/ (10)

5. C - nice so man $\mid \mathrm{him} /(10)$ do the red points

6. A - can't do these ((señala con el dedo a la pantalla))

7. C - why/

8. $\quad$ A - because (4)

9. $\quad \mathrm{C}-(\mathrm{XXX})$ oh/ do that/ (6) o:h man $\backslash$ (13) could you | don't you wann' destroy this/ | destro:y i:t (3) two down | two | (XXX) use it | twice | ah/ don’t des | tro::y it | why didn’t you destro:y/ (4)

10. $\quad \mathrm{A}-$ at least I have (XXX) cards (3)

11. C - yep | I try to beat him but he beat me ((carraspeo)) (2) don't you want 'em making (XXX)

Si bien el fenómeno de cambio de código es menor en este fragmento, el turno 1 parece confirmar lo que venimos diciendo sobre los cambios al español para hablar sobre temas no relacionados con el juego. Por lo menos se sostiene que mientras la lengua base es el inglés, y los interlocutores están hablando de temas relacionados con el juego, la conversación se mantiene en inglés.

Un tercer fragmento de la primera sesión de juegos confirma de alguna manera la tendencia que hemos observado. En esta ocasión el cambio no ratifica que, en casos en que el inglés es la lengua base, se cambia al español cuando se está hablando sobre algo no relacionado con el juego, sino sucede lo contrario: hay un cambio de código al inglés cuando sí se quiere remarcar algo sobre el juego, en este caso, una victoria (ver turnos 2 y 4). 


\section{Fragmento 3}

1. $\quad \mathrm{C}-\mathrm{fo}:: \mathrm{r} \mid \mathrm{I}$ wanted to get $(\mathrm{XXX})$

2. $\quad A-$ vistes/ lo gané $\mid$ y yo no perdí vida te dije lo =gané $\mid$ finally= I win 'em

3. $\quad \mathrm{C}-=$ Ángel $=$ ev... Angel ev... Angel everybody needs his kart ok $\mid$ e:::h everybody choose his kart

4. A - I win

5. C - a:h | lose number four | can I choose number four | d'you know who's (XXX)/ | do you know who's who's number four/ | mar'd it (( voz muy baja)) (5) see mar'd it | your turn (5) u::::h u::h | oh my $\mid$ he took sev... $\mid$ o::h

6. A - a:::h you didn't get it

7. C - I want to my friends get another kart | I don't want o:h | fu:f I'm glad that wet fent didn't go in my seek $\mid=$ oh ma=

8. $\quad \mathrm{A}-=(\mathrm{XXX})=$ but he's so resistant $\mid$ yes/ (2)

9. C - hey look it | hey you just look it | he gets | Mario's | turn | go Mario | uh oh my (7) oh my goodness $\mid \mathrm{a}::$ :h/ he Mario kart has another kart

10. A - a:h (XXX) look at that car that car is so cool (3)

11. $\mathrm{C}-$ what is that for/ (3)

Recapitulando: en la primera sesión, donde el inglés fue la lengua base, el uso de esta lengua tiende a reservarse para hablar sobre aspectos relacionadas con el juego o para regresar a cuestiones del juego cuando se ha cambiado al español; por otro lado, los cambios al español se emplean para referirse a temas no relacionadas con el juego de vídeo, es decir, su orientación es externa. Los cambios de código parecen reforzar esta idea para la mayoría de los casos, si bien puede haber excepciones. Así, parece esbozarse un recurso eficaz de comunicación que permite separar distintas actividades según el idioma empleado: en un caso hablar sobre el juego y en otro referirse a lo que sucede en el entorno.

¿Qué pasa cuando participan otros interlocutores no bilingües? En la segunda sesión de juego la situación ha cambiado. Además de Ángel y Chris, tenemos a sus primos Óscar y Víctor, hablantes nativos del español y con conocimientos limitados del inglés. Recordando que la madre de Ángel y Chris les impuso la consigna de no hablar en inglés mientras estuvieran con otros parientes, la lengua de base es el español. Como veremos, esta consigna entra en juego de manera especial en estas nuevas condiciones comunicativas, modificando el patrón que habíamos registrado en la primera sesión de juego en torno a los cambios de código. 
En el primer fragmento de esta sesión (Fragmento 4) los cambios de una lengua a otra parecen darse por otros motivos. El vídeo comienza con una secuencia en inglés entre Ángel y Chris, quienes se están peleando por algo (turnos 1 y 2). Esta secuencia parece involucrar sólo a los dos hermanos (aunque están rodeados por sus otros primos), y tal vez por eso la llevan a cabo en inglés a pesar de la consigna de la madre. Sin embargo, inmediatamente después (turno 3) Ángel parece recordarla y regresa al español, y a partir de la intervención de Oscar en el turno 4 ya no se regresa al inglés salvo por otros motivos.

Entre estos otros motivos se encuentra lo que podríamos considerar una subdivisión de los cambios de código relacionados directamente con el juego: las inserciones del inglés. Dado que la interacción está regulada por un aparato electrónico, era natural esperar esta forma de cambio, sobre todo considerando que los juegos son producidos y desarrollados en inglés, por lo que resulta prácticamente inevitable emplear palabras inglesas para referirse a lo que sucede en el juego. Es lo que sucede en los turnos 11, 18, 25 y 27 del Fragmento 4, en los que las inserciones aluden a personajes, eventos o al juego mismo, todos en inglés. En los turnos 21, 36 y 37 sucede algo parecido con las inserciones, sólo que son referencias a la cultura popular (un personaje y un juego). Si hemos marcado los nombres propios como casos de esta forma particular de cambio de código es porque la pronunciación de los niños es en inglés, opción que contrasta con la forma en como lo pronunciaría regularmente un niño mexicano.

\section{Fragmento $4^{\text {ii }}$}

1. A - you are mean, you are mean to me

2. C - you are mean to me/

3._A - @@ me pegó, au, au, au, au, au

4. $\mathrm{O}-$ Cristo/

5. $\quad \mathrm{C}-(\mathrm{XXX})$

6. A - uno

7. $\mathrm{O}-$ gracias

8. $\quad \mathrm{C}-(\mathrm{XXX})$

9. A - no necesito más de diez porque ya tengo ciento seis

10. $\mathrm{O}-\mathrm{ah}$, yo tengo sesentisiete y no digo naá

11. A - oh | ve | Stephanie K ((pronunciación inglesa)) y Multi Pica y yo te voy a ganar | porque yo tengo ciento $=$ seis $=$ 


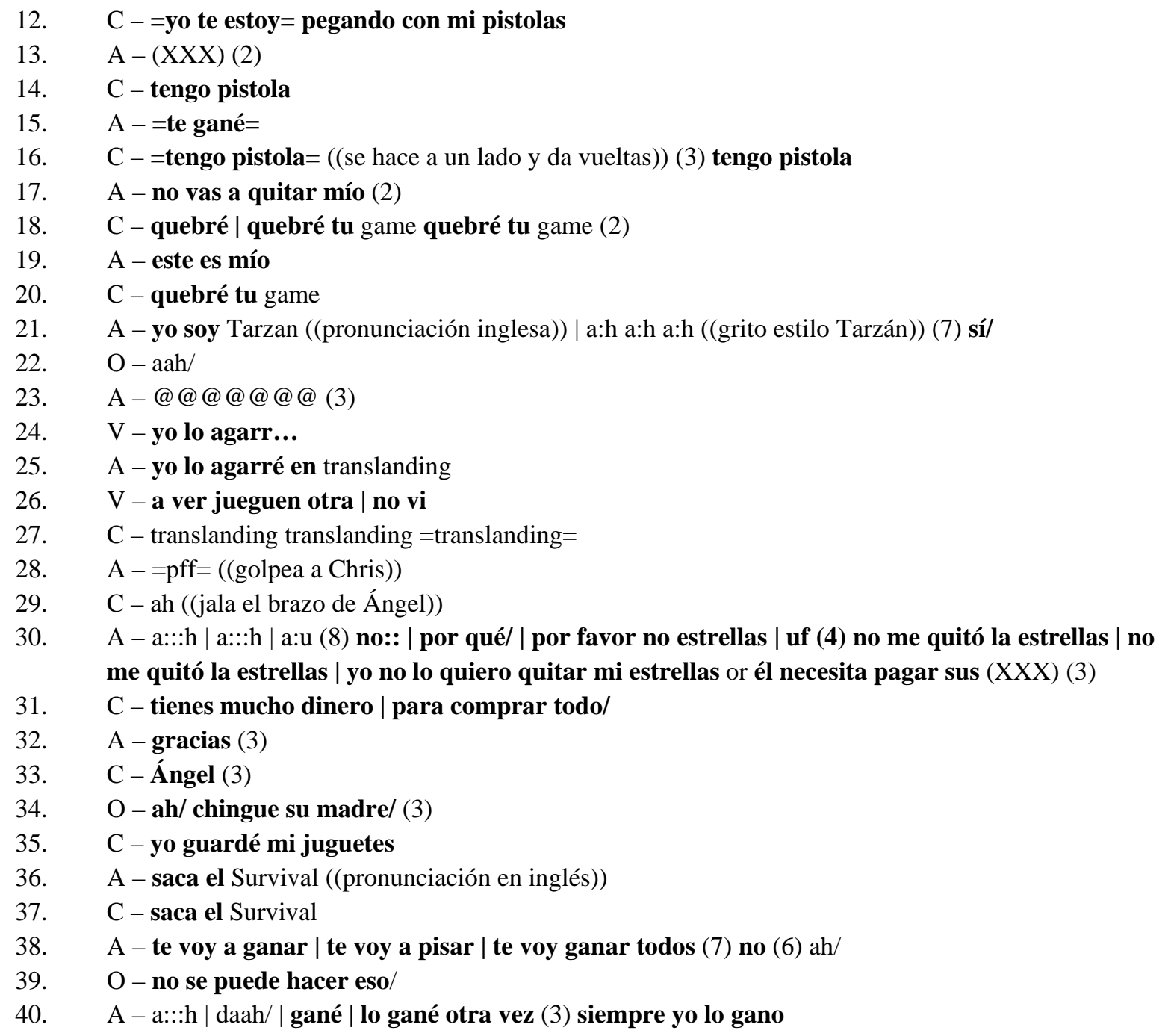

En el siguiente fragmento encontramos datos similares al fragmento anterior. La secuencia comienza con Ángel y Chris hablando entre sí en inglés, como si estuvieran en una conversación aislada, y luego ante la presencia de su primo Oscar recuerdan que deben hablar en español, como se observa en el CS del turno 2. Así parece que el cambio de código sirve para indicar un cambio de situación, en este caso la incorporación de un nuevo interlocutor. No obstante, cuando más adelante hablan sobre elementos relacionados con el juego, parecen regresar al inglés como en la primera sesión de juego (turnos 5 y 6). Finalmente, volvemos a encontrar ejemplos de cambios de código de orientación interna en 
la forma de inserción (turno 9), como cabe esperar en estas situaciones en las que la lengua base es el español.

\section{Fragmento 5}

1. C - hurry/ hurry/

2. $\mathrm{A}-(\mathrm{XXX})$ pick user like $\mid$ no no $\mid$ sí o no

3. $\mathrm{O}-$ chale/

4. A - sí quieres/

5. $\mathrm{C}-$ yeah peros yo no empiezar

6. A - you are cero (5) o:h sí/

7. C - yo no soy cerol

8. $\quad$ A - dah $(\mathrm{XXX})$

9. $\quad \mathrm{C}-\mathbf{o i} \mid$ laser master sis | always (2)

10. A - ah/ me cansé |oye/ continúa/

11.rO-no@@

12. A - oye no estás continuando

13. $\mathrm{O}$ - pérate | quiero leer los dibujos (3)

Recapitulando lo observado en la segunda sesión de juegos, los hermanos Ángel y Chris tienden a recurrir al inglés aun en situaciones en las que cabría esperar el empleo del español (por la consigna de la madre y por funcionalidad comunicativa). Pero basta la participación de otro interlocutor para recordarles que deben hablar en español (resulta interesante que cuando hablan en inglés lo hacen con un volumen muy bajo; el cambio de volumen podría marcar el uso del inglés como una transgresión e indicar la presencia de una norma comunicativa). También encontramos que abundan las inserciones del inglés, las cuales podríamos considerar como una forma particular de cambios de código de orientación interna.

\section{Discusión de los resultados}

Al concluir su análisis del CS observado en las aulas de una escuela barcelonesa entre estudiantes de distintos orígenes étnicos y sociales, Unamuno (2008) cuestiona las teorías que ven una correspondencia rígida entre el estatus social de una lengua determinada y las 
funciones que ejerce en las prácticas interactivas. Para la autora, no existe una relación mecánica entre cambio de lengua y funciones, entre cambio de lengua y contextos. Más bien, estas relaciones deben ser descritas a través de un análisis minucioso de la interacción y ver cómo se definen en cada caso.

En el caso que nos ocupa vemos que suceden ambas cosas. Por un lado ocurre lo que describe Unamuno, en especial en las interacciones correspondientes a la primera sesión de juego. En estos casos, aunque se observan ciertas regularidades en el uso del CS, éstas no son rígidas, lo cual da lugar a las excepciones que mencionamos en el Fragmento 1 durante la negociación de la lengua a utilizar. Aquí los cambios de código parecen constituir un recurso comunicativo: reservar la lengua de base para asuntos relacionados directamente con el juego y cambiar de código cuando se desee hablar sobre hechos o sentimientos ajenos al juego. Los motivos por los que ocurre esto pueden ser diversos, habría que preguntarse qué ventajas comunicativas representa hacer este tipo de separación. Lamentablemente faltó analizar un corpus más amplio y con más ejemplos para poder emitir una hipótesis fuerte. Por lo pronto, para no entrar en aspectos psicológicos, podemos pensar que una razón comunicativa de dichos cambios puede ser precisamente marcar cuándo se está hablando de cierto tipo de tema y cuándo se está hablando de otro. Gumperz (1982) y Auer (1984) señalan que el cambio de lengua, a manera de indicio de contextualización, indica un cambio de tópico conversacional que permite a los interlocutores comprender de forma más precisa la organización de la interacción.

Sin embargo, también hemos observado que existe cierta correspondencia entre el estatus de una lengua y su función en las prácticas interactivas. Ello ocurre en la segunda sesión de juego, en la que la lengua base es el español. Mencionamos que en esta situación el uso de inserciones del inglés (en su mayoría relacionadas con aspectos del videojuego) resulta una práctica habitual, debida principalmente a que la lengua en que se producen y desarrollan los juegos es precisamente el inglés. Desde este punto de vista, el inglés ocupa una relación privilegiada como lengua de creación del juego, en tanto que el español ocupa un puesto secundario porque sus hablantes sólo desempeñan un rol de consumidores, no de 
creadores. En este sentido, muchas veces los actores de nuestro análisis no tienen otra opción más que recurrir al inglés para nombrar ciertas acciones, personajes o el juego mismo, aunque ello implique cambiar de lengua. Su única opción se reduce a la pronunciación (inglesa o española), pero no parecen usar esta distinción como un recurso, sino que eligen la forma que les parece más natural.

Vemos así que el CS en la situación particular que hemos estudiado puede deberse a distintos factores, aunque sin duda se sostiene que representa un recurso más en el repertorio de los hablantes bilingües, motivado por elementos externos e internos del juego. Al formar parte de un repertorio, esto significa que pueden hacer uso de los recursos de manera estratégica o con cierto sentido, como se ha mostrado en el caso de Ángel y Chris.

Con esto también coinciden Bauer y colaboradoras en su estudio sobre el juego en niños bilingües, al afirmar que las sutiles diferencias en la forma en que la niña estudiada usa sus dos lenguas sugieren que, mediante su interacción con distintos interlocutores durante el juego, estaba aprendiendo y ensayando distintas habilidades comunicativas en ambas lenguas. De manera particular, estaba aprendiendo cómo el idioma que aprendía influía en las acciones de su interlocutor. Lo mismo puede decirse de Ángel y Chris, quienes paulatinamente van aprendiendo qué pueden decir en español en determinado contexto (que en ocasiones estará acotado, como en este caso) y qué pueden decir en inglés en otras situaciones, y manejarlo según convenga a sus intereses comunicativos. Esto sin mencionar el vínculo que generan al poder diferenciarse de otros niños y otras familias que no tienen la oportunidad de interactuar en dos idiomas de manera tan fluida.

Los resultados de nuestro estudio de caso también coinciden con los del caso de Bauer (2002), al reiterar que el ser criado en dos idiomas no produce necesariamente competencias equilibradas en ambas lenguas. En estos casos conviene recurrir a la noción de competencia plurilingüe (Unamuno 2008; Pekarek Doehler 2006; Moore 2006; Lüdi \& Py 2009): el plurilingüismo entraña competencias dispares entre las distintas lenguas, pero también conlleva el desarrollo de habilidades que permiten adecuar y gestionar los repertorios lingüísticos al contexto, tomando en cuenta interlocutores, actividades y temas. 


\section{Conclusiones}

Al comenzar este estudio sobre la relación del CS con los juegos de vídeo pensábamos que, dadas las características del contexto comunicativo y los participantes, las secuencias y los cambios de código estarían orientados más directamente hacia el juego (sobre todo por las inserciones del inglés), si no de manera exclusiva, sí en una elevada proporción. Sin embargo, encontramos que buena parte de los cambios de código se debían a factores externos al juego. Así, en la primera sesión de juego detectamos una tendencia a cambiar de idioma (de inglés a español) cuando los participantes querían comunicar algo no perteneciente al juego en curso. No obstante, encontramos algunas excepciones, confirmando que en cuestiones relativas al lenguaje la búsqueda de patrones mecánicos suele ser infructífera. Aún así podemos aventurarnos a hacer algunas afirmaciones, por ejemplo, que los niños emplearon el cambio de código como un recurso que indica modificaciones en la configuración de los interlocutores (como se ve en la sesión de juegos en la que participan sus primos), en la disponibilidad léxica (palabras que sólo estaban disponibles en inglés) y cambios de tema (para señalar que se estaba hablando de asuntos relativos al juego o al entorno).

Futuras investigaciones deberán incluir forzosamente un corpus más amplio, tanto en el número de participantes como en la extensión de las conversaciones grabadas y transcritas, a fin de generalizar más ampliamente las tendencias registradas. La dificultad radica en encontrar material en contextos similares a los que presentamos, ya que no todo investigador tiene acceso a conversaciones en la intimidad de unas vacaciones familiares, en especial si los sujetos a estudiar son niños. En la medida de lo posible, los investigadores deberán tratar de comprender los videojuegos en los que estén participando los niños, ya que esto no nos fue posible en esta ocasión, dificultando en gran medida la interpretación de lo que se dijo durante la interacción. Además de registrar en vídeo la interacción de los hablantes, como hemos hecho, lo ideal sería también grabar lo que sucede en pantalla para poder relacionar mejor las emisiones de los interlocutores con 
algún recurso comunicativo particular. Ello implicaría disponer de dos cámaras a la vez, lo cual puede resultar una dificultad técnica.

Pero a pesar de las limitaciones de este estudio, consideramos que sienta un antecedente en este territorio poco explorado de la interacción de niños bilingües jugando videojuegos. La información que generen estudios complementarios permitirá comprender mejor cómo interactúan los niños en distintos contextos, cómo aprenden a utilizar su lengua o lenguas, y qué recursos utilizan para producir significados en nuevas situaciones. Los videojuegos, de uso creciente y generalizado entre niños y jóvenes, aún no son objeto de consideración escolar. Sin embargo, tal y como sostiene Gee (2004), son contextos de aprendizaje que los investigadores deben considerar a la hora de preguntarse qué aprenden los niños. En este sentido complementan la didáctica de las lenguas y arrojan luz sobre el modo de llevar a cabo diferentes tareas.

\section{Referencias bibliográficas}

Álvarez-Caccamo, C. (1998) From 'switching code' to 'code-switching': Towards a reconceptualisation of communicative codes. En: Auer, P. (1998). Code-switching in Conversation, pp. 29-48. London: Routledge.

Ardila, A. (2005) Spanglish: An Anglicized Spanish Dialect. Hispanic Journal of Behavioral Sciences; 27; 60.

Auer, P. (1984) Bilingual conversation. Amsterdam: Benjamins.

Bauer, E. B. (2000) Codeswitching during shared and emergent reading: Lessons learned from a preschooler. Research in the Teaching of English, 35(1), 101- 130.

Bauer, E.B., Hall, J.K. y Kruth, K. (2002) The pragmatic role of codeswitching in play contexts, International Journal of Bilingualism, 6; 53.

Cromdal, J. (2005) Bilingual order in collaborative word processing: on creating an English text in Swedish. Journal of Pragmatics, 37, 329-353.

Gee, J.P. (2004) Lo que nos enseñan los videojuegos sobre el aprendizaje y el alfabetismo. Málaga: Ediciones Aljibe.

Genesee, F., Nicoladis, E. y Paradis, J. (1995). Language differentiation in early bilingual development. Journal of Child language, 22, 611-631.

Grosjean, F. (1989) Neurolinguists, beware! The bilingual is not two monolinguals in one person. Brain and Language, 36, 3- 15. 
Gumperz, J. J. (1982) Conversational code switching. En J. J. Gumperz (Ed.), Discourse strategies, pp. 59-100. Cambridge, U.K.: Cambridge University Press.

Gumperz, J. J.y Hymes, D. (Eds.). (1972) Directions in sociolinguistics. The ethnography of communication. NewYork: Holt, Rinehart\&Winston.

Liebscher, L. y Dailey-O’Cain, J. (2005) Learner code-switching in the content-based foreign language classroom. The Modern Language Journal, 89, 234-247.

Lüdi G. y Py B. (2009) To be or not to be... a plurilingual speaker. International Journal of Multilingualism. 6(2):154-167.

Lüdi, G. y Py, B. (2003). Etre bilingue. 3a ed. Bern, New York: Peter Lang.

Mondada L. (2007) Le code-switching comme ressource pour l'organisation de la paroleen-interaction, Journal of language contact - THEMA 1: 168-197.

Moore, D. (2006) Plurilingüismes et école. Paris: Didier/LAL: 77-113.

Nicoladis, E. y Genesee, F. (1996) A longitudinal study of pragmatic differentiation in young bilingual children. Language Learning, 46 : 439- 464.

Nussbaum, L. (2001) El discurso en el aula de lenguas extranjeras. En L. Nussbaum y M. Bernaus (eds) Didáctica de la lengua extranjera en la ESO, pp. 137-172. Madrid. Síntesis.

Nussbaum, L. y Tusón, A. (2003) ¿Existe una lengua base en la conversación bilingüe?. Actas do I Simposio Internacional sobre o Bilingüismo. Santiago de Compostela.

Pekarek Doehler, S. (2006) Compétence et langage en action. Bulletin suisse de linguistique appliqué, 84 : 9-45.

Unamuno V. (2008) Multilingual switch in peer classroom interaction. Linguistics and Education 19: 1-19.

Unamuno, V. (2004) Cuando las lenguas se encuentran. Algunos problemas empíricos. En: A. Raiter, J. Zullo, V. Unamuno, (Eds.). Sujetos de la lengua: introducción a la lingüística del uso, pp. 137-161. Barcelona: Gedisa.

Zentella, A.C. (1981) Ta bien, you could answer me en cualquier idioma, Puerto Rican codeswitching in bilingual classrooms. En R. Duran (Ed.), Latin languages and communicative behaviour, pp. 109-132. Norwood: Ablex Press.

\section{Apéndice 1}

\section{Glosario de símbolos utilizados}

(( )) Fenómenos simultáneos al habla (sonoro, extraverbal, paraverbal, etc.)

| Pausa breve

(3) Pausa prolongada (duración en segundos)

/ Entonación ascendente 


\begin{tabular}{ll}
$\backslash$ & Entonación descendente \\
$:$ & Alargamiento de sonido \\
$(\mathrm{XXX})$ & Ininteligible \\
$\ldots$ & Interrupción \\
$@$ & Risa \\
$==$ & Solapamiento \\
Negritas & Enunciaciones en español \\
\hline
\end{tabular}

\section{Referencia del autor:}

Armando Robles Hmilowicz es psicólogo organizacional por el Instituto Tecnológico y de Estudios Superiores de Monterrey y filósofo por la Universidad Nacional Autónoma de México. Desde hace cuatro años es traductor y editor free-lance para distintas editoriales en México y Argentina. Actualmente estudia la maestría en Análisis del Discurso en la Universidad de Buenos Aires.

\section{Email: arobles77@yahoo.com}

1 La distinción ortográfica “Angel”/“Ángel” (sin tilde y con tilde) obedece a la pronunciación: “Angel” corresponde a la pronunciación inglesa mientras que "Ángel” a la española.

2 En el Fragmento 4 Ángel y Oscar están jugando el juego de vídeo Mario Kart, cada uno en una consola individual, mientras que Chris y Víctor juegan a las pistolas alrededor de Ángel y Oscar. En ocasiones Ángel y Oscar hablan entre sí o con la máquina sobre el juego y en otras hablan a Chris y Víctor. 